\title{
Reconfiguración del campo mundial de las universidades: transformaciones en los procesos contables y de la gestión universitaria
}

Juan David Cardona-Hernández

Universidad de Antioquia

Colombia 


\title{
Reconfiguración del campo mundial de las universidades: transformaciones en los procesos contables y de la gestión universitaria ${ }^{1}$
}

\author{
Juan David Cardona-Hernández
}

Universidad de Antioquia - Colombia

Para citaciones: Cardona Hernández, J. (2021). Reconfiguración del campo mundial de las universidades: transformaciones en los procesos contables y de la gestión universitaria. Panorama Económico, 29(2), 84101.

Recibido: 10 de noviembre de 2020

Aprobado: 15 de marzo de 2021

Autor de correspondencia: Juan David Cardona-Hernández jdcardonah@yahoo.es

Editor: Andrés Escobar E. Universidad de Cartagena-Colombia.

Tipología IBN Publindex: Artículo de Reflexión

Copyright: (C) 2021. Cardona Hernández, J. Este es un artículo de acceso abierto, distribuido bajo los términos de la licencia https://creativecommons.org/licenses/bync-sa/4.0/ la cual permite el uso sin restricciones, distribución y reproducción en cualquier medio, siempre y cuando que el original, el autor y la fuente sean acreditados.

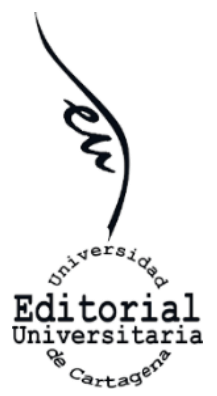

\section{RESUMEN}

Los cambios suscitados en el campo mundial de la economía han impactado de diversos modos a las universidades, y en el contexto del neoliberalismo y la Nueva Gestión Pública, la racionalidad empresarial empieza a incardinarse en diversos espacios de la vida, tanto en lo que se refiere al gobierno de sí, como de instituciones que eran lejanas a una racionalidad de este tipo. Un ejemplo de eso se manifiesta cuando dicha racionalidad llega al mundo de las universidades, lo que conlleva a la incorporación de valores, lenguajes y redes de prácticas que transmutan el sentido de dichos espacios de educación superior. La racionalidad de antaño, guiada principalmente por el capital cultural migra para darle paso a la universidad empresarial, que acoge de manera novedosa procesos contables coherentes con esa nueva racionalidad económica. Es así, como los presupuestos, los costos y la auditoría ganan terreno bajo preceptos cada vez más financieros para la toma de decisiones, convirtiéndose así en un foco para el control de las dinámicas institucionales, como para guiar las conductas en los sentidos que esa nueva racionalidad lleva consigo. A partir de una revisión documental, se problematizan los cambios que han tenido las universidades, mostrando cómo los cambios de racionalidad y del rol de los sistemas contables, generan redes de prácticas que pervierten el sentido de la universidad hacia fines principalmente económicos incorporando una racionalidad instrumental en su gestión que desdice de los fines que la universidad comprometida con el conocimiento y la sociedad.

Palabras clave: Universidad; contabilidad; auditoría; neoliberalismo; Nueva Gestión Pública.

\section{Reconfiguration in the world management of universities: transformations in accounting and university management processes}

\section{ABSTRACT}

The global changes in the economy have impacted universities in various ways. In the context of neoliberalism and New Public Management, business rationality begins to get involved in various areas of life, such as, the government and other institutions that were far from a rationality of this type. One of those institutions are precisely universities. The commented rationality entails the incorporation of values, languages and networks of practices that convey the meaning of such higher education institutions. The rationality of yesteryear, guided mainly by cultural capital, migrates to make way to the business university, which welcomes accounting processes consistent with this new economic rationality in a novel way. Thus, budgets, costs, and auditing gain ground

${ }^{1}$ El presente artículo de investigación forma parte del proyecto de investigación: "La planeación universitaria como tecnología de gubernamentalidad en un campo de disputa: Un análisis desde Pierre Bordieu y Michel Foucault de la construcción de las bases estratégicas del Plan de Desarrollo 2017-2027 de la Universidad de Antioquia", el cual fue presentado para optar al título de Magister en Sociología de la Universidad de Antioquia. 
under increasingly financial precepts for decision-making, becoming a focus both for the control of institutional dynamics, and for guiding the behaviors that this new rationality brings with it. Based on a documentary review, the changes that universities have undergone are problematized, showing how changes in rationality and the role of accounting systems generate networks of practices that pervert the meaning of the university, leading them towards mainly economic ends. It is observed that the incorporation of an instrumental rationality in university management belies the aims of the university committed to knowledge and society.

Keywords: University, accounting, auditing, control, neoliberalism, New Public Management.

\section{INTRODUCCIÓN}

La universidad es una institución antiquísima, que surge en la transición entre la premodernidad y la modernidad, pero que se va a convertir en el bastión del espíritu moderno, y en el epicentro de la difusión y construcción de conocimiento y cultura. La universidad que en sus inicios estaba muy arraigada a la teología, iba verse luego enfrentada a los embates de la época y los discursos del pensamiento científico y racional comienzan a atravesarla. Ya no será entonces el lugar de la fe, sino de la razón, y es allí donde el ser mismo de la universidad transmuta.

Han pasado siglos desde que las primeras instituciones universitarias vieron su origen en la Europa medieval, y los problemas que las atraviesan han mutado también. A la discusión por la docencia, la investigación y la extensión, se le suman una plétora de discursos que enfatizan en los procesos de gestión universitaria, que como ejercicio de problematización, en el sentido Foucaultiano ${ }^{2}$, significa traer a escena preguntas, pero también respuestas sobre lo que debe ser la gestión de las universidades.

Discursos que van desde el plano internacional, nacional y local, que catapultan y dan sentido a prácticas institucionales que se conectan de manera escalar con los discursos hegemónicos sobre la gestión universitaria.

Reflexionar sobre lo anterior significaría conectar acontecimientos disímiles de relaciones que son a la vez globales y locales, donde los vínculos no surgen de una necesidad, sino de acontecimientos que se conectan en un orden no necesariamente predecible y con consecuencias diversas en el espacio social; por lo tanto, para quienes desean pensar la universidad, se hace necesario el ejercicio genealógico ${ }^{3}$, y más aún cuando se trata de relacionar aspectos que en el contexto se encadenan con los procesos nacionales y locales, y que tienen eco en los procesos universitarios, que si bien tienen una autonomía

\footnotetext{
${ }^{2}$ Para Foucault, plantea Sauquillo, la problematización está asociada directamente con los juegos de verdad, y hace parte de los procesos de historización de los discursos (en el sentido arqueológico) y las técnicas de gobierno (en el sentido genealógico). Dirá dicho autor que Foucault entiende por problematización "el conjunto de prácticas discursivas o no discursivas -prácticas institucionales y aparatos de conocimiento-, configurador de los juegos de lo verdadero y de lo falso, por el que algo emerge como objeto de reflexión moral, conocimiento científico, o análisis político" (Sauquillo, 2001, p. 81).

${ }^{3}$ Dirá Núñez en relación al ejercicio genealógico que el "papel del genealogista consiste en buscar a qué tácticas, dispositivos y fuerzas responden los saberes científicos y las instituciones vigentes, además de identificar también las tácticas y las fuerzas a la que responden los saberes de la gente" (2011, p. 79).
} 
relativa, no están exentos de los impactos en los cambios que en el espacio social acontecen ${ }^{4}$.

Metodológicamente, el presente trabajo se fundamenta en una revisión de literatura que permitiera problematizar el contexto de las universidades en relación al neoliberalismo y los procesos globales que han implicado cambios en la gestión de las Universidades. El presente ejercicio reflexivo no pretende caer en el estructuralismo que subsume la realidad bajo un manto determinista, más bien busca dar unos elementos generales, marcando tendencias importantes en los procesos que impelen a las universidades a adoptar cierta racionalidad. Más allá de la homogeneidad, el marco de análisis no puede olvidar que son las prácticas concretas de las organizaciones las que permiten evidenciar el desdoblamiento de las políticas y lineamientos globales, donde hay procesos de adaptación, pero también procesos de resistencias, y ciertos amalgamamientos inéditos producto de las tensiones a nivel organizacional y de las luchas en el campo de las universidades.

En coherencia con lo anterior, el presente trabajo busca dar cuenta de las condiciones de posibilidad para los procesos de cambio en la racionalidad administrativa de la universidad. Para ello se presenta inicialmente cómo los procesos de globalización tienen un impacto en la gestión de las universidades, donde una suerte de discursos administrativos empresariales llega desde lo global a incidir en el campo de las universidades. Posteriormente, se presentan los cambios discursivos en el ámbito de la gestión, cómo allí se empiezan a concretar enunciados que van más de la mano con la incorporación de una discursividad proveniente de la empresa privada. Finalmente, se presentan las principales conclusiones.

\section{GLOBALIZACIÓN NEOLIBERAL Y EL ARRAIGO DE LA FORMA EMPRESA EN LA UNIVERSIDAD PÚBLICA}

Comprender los cambios en la gestión universitaria y en particular en los procesos de planeación en la Universidad, implica una mirada crítica de los procesos históricos acaecidos en el campo de las universidades a partir de las transmutaciones nuestra época.

Esto, en sintonía con el llamado que hace Vega quien manifiesta que en nuestra época:

"un análisis serio y riguroso de las transformaciones de la universidad no puede hacerse al margen de las modificaciones del capitalismo ni de la implantación de la lógica mercantil" (2015, p. 4).

Pensar la universidad hoy significa entonces pensar el marco en el cual se ha venido transformando las dinámicas societales en la globalización, donde el contexto mundial es propicio para que las lógicas mercantiles ingresen en ámbitos en los que antes no tenía tanta preponderancia.

\footnotetext{
${ }^{4}$ Dirá Alicia Gutiérrez al respecto: "Hablar de autonomía relativa supone pues, analizar las prácticas en el sistema de relaciones específicas en que están insertas, es decir, según las leyes de juego propias de cada campo, leyes que mediatizan la influencia de los demás espacios de juego, pero supone también la presencia de los demás campos que coexisten en el espacio social global, cada uno de ellos ejerciendo su propia fuerza, en relación con su peso específico" (En Bourdieu, 2013, p. 14).
} 
Entender lo local en el entramado de lo global se torna pues en un reto fundamental, para no caer por un lado en el imperialismo de la estructura donde lo global se impone como fuerza arrasadora que determina todo lo que toca como una suerte de nuevo rey Midas.

Ni caer tampoco en el imperialismo del individuo o las organizaciones desconociendo los contextos específicos en que ciertas relaciones son posibles, mientras otras se constriñen. Más bien se trataría de una dialéctica.

Hoy en día la sociedad de alguna manera es local, nacional, regional, y global de forma simultánea, en cuanto experiencia, política, efectos e imaginarios. Además, estos espacios se superponen a las relaciones de poder desiguales que reflejan la geopolítica contemporánea y luchas políticas pasadas. Las geografías del poder residuales, dominantes, emergentes y rebatidas, incluyendo las del pasado colonial y el presente poscolonial, están en juego en todos los espacios globales y se manifiestan de forma vernacular en lo local, nacional y regional (Rivzi y Lingard, 2013, p. 93).

En concordancia, cabe resaltar que lo que se presenta en este apartado son tendencias generales, lo que no significa una determinación unívoca, por el contrario, se pretende señalar que estas dinámicas generales y tendencias pueden servir de marco de interpretación de prácticas locales, posibilitando establecer conexiones de lo global con lo local, pero también ubicar puntos de deslinde.

Se destaca entonces la pluralidad de la realidad social, y en ese sentido, se resalta la idea de que es el trabajo de campo específico de las realidades concretas de las universidades lo que permitirá afirmar si una u otra universidad siguen la tendencia general aquí marcada, o por el contrario hay puntos de ruptura con ese contexto global.

Teniendo lo anterior como referente, vale decir que la universidad se ha encaminado a procesos en los que se fusionan de manera singular el campo de la educación con su racionalidad, y el campo económico, campos que en la actualidad convergen en espacios de relacionamiento globales. Como señala Leopoldo Múnera, la universidad de antaño, la universidad liberal que se había conocido "es relegada a un pasado que debe ser superado, en virtud de la adaptación funcional y acrítica al presente de la globalización" (2005).

Los procesos económicos y sociales están cada vez más interconectados a escala mundial, la internacionalización se vuelve parte integrante de las agendas universitarias. Los intercambios institucionales de docentes y estudiantes toman fuerza, los convenios son mayores entre universidades de diferentes latitudes, las comparaciones y competencias se evidencian en la lucha por el capital simbólico en los rankings internacionales, los procesos de estandarización de currículos y homologación de títulos, acreditaciones nacionales y globales, empiezan a ser un imperativo la movilidad académica, entre otros aspectos. 


\section{IMPACTO DEL CAMBIO EN LA RACIONALIDAD ORGANIZACIONAL DE LO PÚBLICO CASO: Cambios en el sistema de salud de Nueva Zelanda}

En el caso de los cambios en el sistema de salud de Nueva Zelanda el estudio de Lawrence, Northcott y Lowe (1997) es un ejemplo relevante del papel de que juegan los sistemas contables administrativos en las organizaciones. Los autores evidencian, para el caso de la salud cómo al incorporar la racionalidad que define la prestación del servicio de salud por preceptos de rentabilidad y de mercado, el actuar del sistema de salud se transforma. Dirán al respecto Barrios y Rivera (2008)

antes de la reforma, la premisa básica de la prestación de los servicios de salud, era atender las necesidades de todas las personas independientemente de su capacidad de pago y del costo del tratamiento. La salud era un servicio público. Los profesionales médicos tenían un gran poder y tomaban sus decisiones basados en su saber experto, siendo el factor monetario un asunto secundario.

No obstante, tras la reforma, se sujetó la prestación de los servicios de la salud al sistema de precios del mercado. La forma de ver las clínicas cambió a partir de entonces, ya no significaban entidades de asistencia social sino "negocios" que debían ser "exitosos" y ofrecer sus productos en un mercado competitivo. La asignación de los recursos aquí, no se hace por distribución presupuestaria, sino con base en los resultados de todas las unidades del hospital, medidas por el número de pacientes e ingresos recibidos.

En estos cambios, los sistemas contables jugaron un papel importante, porque entregaban información útil acerca de todos los costos incurridos por el hospital: los salarios de los cirujanos, los medicamentos y materiales quirúrgicos, la permanencia de un paciente, permitiendo al tiempo la tarificación de los servicios prestados, información no requerida antes de la reforma, dado que todo paciente debía atenderse independiente del costo de su tratamiento (p. 66-67)

Todos estos cambios implicaron no sólo nuevos sistemas contables, sino también un nuevo redimensionamiento de las prácticas y del quehacer, pues la racionalidad económica implicaba nuevas formas de trabajo, atención a los pacientes y utilización de recursos. Los médicos pierden protagonismo en las decisiones, para cedérselo a expertos en finanzas. "Los médicos cedieron poco a poco su poder. Su saber experto fue socializado y traducido al lenguaje de la contabilidad y la economía, y el acceso a los recursos condicionado a su desempeño económico" (Barrios y Rivera, 2008, p. 67).

Pero de la mano a la configuración del campo internacional de las universidades, el capital económico gana relevancia ${ }^{5}$ en su dinamización. Diversos organismos internacionales insisten el hacer converger cada vez más la educación a los circuitos comerciales (Vega, 2015; Martínez, 2014; Galcerán, 2010; Pelayes, 2000; Feldfeber, 2009). Lo anterior, señalaría cambios en los que se vería afectado, y redimensionado el campo universitario, en donde su autonomía (relativa) se vería afectada por el campo económico mundial, en donde la educación en la globalización, parafraseando a Bourdieu, es considerada como una mercancía cualquiera, y, por tanto, tratada como cualquier producto, y sometida a la ley del beneficio (2001, p. 83).

\footnotetext{
${ }^{5}$ Se destaca la idea de la configuración del campo internacional de las universidades, en la cual el capital cultural sigue operando como dinamizador de las luchas en el campo. No se trata, como diría Appel de que "se experimenta la translación de la educación de la esfera pública a la privada, lo que se concreta en el tránsito de la universidad como institución de capital cultural a institución de capital económico" (1995, p. 103. En Audelo, 2013, p. 23). Aquí, se sostiene que la irrupción con más fuerza del campo económico al campo universitario significa una reconfiguración del campo, y el peso e importancia de los capitales en los juegos transaccionales, pero esto no significa que el capital cultural haya dejado su papel en las luchas al interior del campo. Frente a la importancia mayor que adquiere el capital económico en el campo de las universidades, el campo se reconfigura, y los productos y relaciones se tornan diferentes en relación a las disputas del capital cultural. Hay otros sentidos y otros requerimientos, pero en el marco de las disputas al interior del campo que son tanto disputas por el capital cultural, como simbólico y económico.
} 
Ese mayor entrelazamiento del campo universitario con el campo económico, en particular el subcampo de las universidades públicas, no significa la pérdida absoluta de la autonomía del campo universitario, en el sentido de que la universidad se rija necesariamente por una racionalidad calcada tal cual del mundo empresarial privado con fines de lucro, sino que se da un redireccionamiento, y las lógicas de la docencia, la investigación y la extensión, empiezan a mutar y a adquirir otro sentido. En términos de los límites que la perspectiva mercantil de la educación plantea, Soforcada (2009) señala:

Los actuales procesos de privatización y mercantilización implican, al igual que tres siglos atrás, tanto el cercamiento del conocimiento y de los bienes culturales, como el cercamiento de la posibilidad de participar en ellos y, en definitiva, el cercamiento de lo público. Esto afecta el conocimiento en sí, en la medida en que pasa a ser concebido en términos de bien comercial, pero también su producción, circulación y transferencia (p. 370).

De esto que no sea extraño que la educación y la cultura constituyan algunos servicios incluidos en el Acuerdo General sobre el Comercio de Servicios (AGCS) de la Organización Mundial del Comercio y otros tratados comerciales regionales (Soforcada, 2009, p. 364).

Todo esto va de la mano con el mayor protagonismo de las universidades privadas $6, y$ por la concepción en la cual la educación pública o privada deben acoplarse a una suerte de isomorfismo con las de la empresa privada, incorporando su visión del mundo (de la economía de mercado y la liberalización), su racionalidad, aflorando prácticas que sin ser exactamente igual al de una empresa capitalista, busca converger con ellas y apropiárselas en el discurso como en sus formas de gestión. En ese orden de ideas, ya no se trata sólo "de la participación del sector privado en la provisión del servicio, sino también de configurar un mercado donde no lo había o de instalar una racionalidad propia del mismo en cuestiones históricamente pensadas en términos del bien social" (Soforcada, 2009, p. 364).

En correlato, parte de toda estos cambios están asociados a lo que diversos autores han denominado la racionalidad neoliberal, la cual implicó, tanto cambios en la comercialización de mercancías a nivel mundial, como la posibilidad de integración de mercados financieros y la transferencias de capitales a diferentes latitudes, división internacional del trabajo y del conocimiento más marcada, la compartimentación de la producción según las necesidades del capital difuminada en diferentes países del globo, la globalización de las políticas macroeconómicas (a cargo de organismos transnacionales como el FMI, el BM, la OMC, la OCDE, la UNESCO, entre otros), procesos de comunicación más interconectados a escala mundial, y revolución en el transporte de mercancías y de personas (Lotta, 2004).

El neoliberalismo, en términos de Rivzi y Lingard (2013) se fundamenta:

\footnotetext{
${ }^{6}$ Un ejemplo de lo anterior son los procesos de privatización de la educación han tenido un ascenso acelerado. En América Latina, las universidades pasaron de 812 (60.7\% privadas) en 1995 a 1.213 (69.2\% privadas) en 2002 (López, 2009, p. 257). En cuanto a las matrículas de las universidades, estas pasaron de 5.070 .731 estudiantes (30.4\% en privadas) en 1995 a 8.316 .649 (40.1\% en privadas) en 2002 (López, 2009, p. 257). Para Colombia el panorama no es muy distinto y la proporción entre universidades públicas y privadas no es muy distante del de América Latina. Éste país tiene, según el Ministerio de Educación Nacional 81 Universidades, de las cuales 31 (38\%) son de carácter oficial o públicas, 49 (61\%) son no oficiales o privadas y una universidad de régimen especial.
} 
en una naturalización dominante de las lógicas del mercado y lo justifica basándose en la eficiencia e incluso la "equidad". Enfatiza el concepto de elección y los privilegios de un gobierno con escaso número de funcionarios, privatización, liberalización y regímenes competitivos de distribución de recursos frente al concepto de Estado Centralizado. Pone énfasis en los regímenes globales de "libre comercio", y los aplica a bienes y servicios, incluso a servicios como la salud y la educación que fueron tradicionalmente marcados por su carácter nacional (p. 58).

Para estos autores, el neoliberalismo, más que políticas económicas y procesos de mercado constituye una racionalidad que va tomando fuerza y se instaura en la subjetividad de las personas y en las dinámicas organizacionales.

En esta misma dirección Castro Gómez (2010) al hablar de la racionalidad del neoliberalismo Norteamericano, desde los planteamientos de Foucault (2008), manifiesta que éste trata de extender la forma-empresa hacia todos los ámbitos de la vida, pues "Sólo de este modo la responsabilidad y credibilidad moral que debe regir el mundo de los negocios se extenderá hacia otros ámbitos, como la familia, la educación, la política, el trabajo, etc." (Castro-Gómez, 2010, p. 201)

Esta racionalidad a su manera y con sus singularidades van a repercutir en las universidades, en las cuales sus funciones misionales son trastocadas y se dinamizan de maneras diferentes, con mixturas nunca antes vistas y concatenadas a las lógicas del mercado con mayor fuerza que antes. No se trata entonces, como se señalaba antes, sólo de que la educación se trance como una mercancía, sino que la racionalidad de la empresa se incorpora en las prácticas organizacionales y de gestión tanto en las universidades públicas como privadas.

Entre los impactos que generó el neoliberalismo económico se encuentra lo que Boaventura de Sousa Santos llama la Crisis Institucional, la cual es "resultado de la contradicción entre la reivindicación de la autonomía en la definición de valores y objetivos de la universidad y la presión creciente para someterla a criterios de la eficiencia y la productividad de naturaleza empresarial o de responsabilidad social" (Santos, 2007, p. 22). Como era de esperarse, todos estos cambios afectaron la dinámica institucional, pues de la mano de una buena gestión y austeridad con los recursos, habría que tomar medidas que pusieran a tono la Universidad con las realidades del mercado y la internacionalización, lo que en otras palabras se tradujo en relación a los aspectos misionales en una transformación

de las actividades de las universidades en servicios mercantiles, así adquiere creciente importancia en las universidades públicas aquellas actividades que sirven para ganar clientes y generar ingresos. De esta manera, secuestrada su esencia por la visión mercantil, las universidades procuran resolver sus dificultades financieras solucionando problemas a las empresas de capital privado, con lo que se establecen, entre universidades públicas y privadas, profusas relaciones mercantiles que terminan por pervertir las funciones sustantivas de la universidad (Ornelas, 2009 p. 100).

En esa misma dirección, la investigación en las universidades termina remplazando los departamentos de investigación y desarrollo industriales, donde los productos del conocimiento derivados de las primeras terminan siendo apropiados y patentados por 
las segundas (Sibilia, 2005, p. 110). La universidad termina estando al servicio del capital privado, que, bajo el manto de la financiación a la investigación universitaria, se apropia de conocimientos construidos por docentes de universidades públicas y privadas. El lucro, termina siendo un criterio relevante para el desarrollo de una determinada investigación. Para Múnera, se dará el "nacimiento de una nueva universidad. Una suerte de fábrica del conocimiento útil, coyunturalmente flexible de acuerdo con la demanda, funcional y acrítica, evaluada permanentemente en términos de su eficacia y rentabilidad económicas" (2005).

De todo lo anterior, que los sistemas de gestión no podían ser los mismos ante los retos que se avizoraban para las universidades, había que hacerles frente a las exigencias de un mundo cada vez más globalizado y bajo estos preceptos la administración universitaria debía mutar para darle paso a los requerimientos de información y control que traían consigo todos estos cambios. Como señala Francisco López, la crisis institucional a la que se refería Boaventura de Sousa Santos lleva a que debido a la presión de las demandas del mercado tanto a las universidades públicas y privadas se administren y funcionen como a una empresa (López, 2009, p. 259).

Las decisiones en la universidad deben ser entonces racionalizadas bajo la lógica de la eficiencia, la eficacia, en busca de minimizar costos y maximizar la obtención de beneficios financieros por diferentes medios. Esto, llevó a que la racionalidad empresarial en gestión y la rendición de informes empezaran a jugar un papel más relevante en las dinámicas universitarias, pues se convertía en el tamiz bajo el cual se podría evaluar no sólo la productividad institucional, sino los resultados, consignados en los planes de desarrollo y de acción en objetivos, metas e indicadores de las Universidades.

\section{CAMBIOS EN LA GESTIÓN UNIVERSITARIA EN LA GLOBALIZACIÓN NEOLIBERAL}

El contexto del neoliberalismo y de las reformas del Estado, se dio lo que diversos autores denominan el New Public Management ${ }^{7}$, el cual acarrearía un nuevo sentido del papel del Estado y de lo público. Según esta concepción de la gestión estatal, se debía "introducir el "Espíritu empresarial" en la cultura de la Administración Pública" (Chica, 2011, p. 60). En el caso de las universidades públicas, esto conllevaría a la "irrupción de los valores provenientes del mundo de los negocios al ámbito académico" (Audelo, 2013, p. 13). "El new management conquista la gestión de las universidades: en donde el aumento de la productividad, ahorro de costes y competencia entre instituciones son claves en la bóveda del nuevo modelo" (Ferreiro, 2010, p. 120).

\footnotetext{
${ }^{7}$ El modelo de la Nueva Gestión Pública "se adoptó principalmente en el Reino Unido y Estados Unidos y se extendió a muchos países deseosos de encontrar soluciones adecuadas para hacer frente a las problemáticas de la gestión pública" (Girotto, Mundet, Llinás, 2013, p. 99). Es de entender, que el New Public Management no es producto como tal del neoliberalismo, el cual se basa más en los procesos globales de privatización, aperturas de mercados para las mercancías y el capital, y en general, siguiendo a Foucault, una racionalidad que se instala, buscando que la forma empresa se instale en las subjetividades de los agentes o grupos de agentes, se institucionalice y se incorpore a organizaciones que no son necesariamente de cuño empresarial privado. La nueva Gestión Pública, no pretende la privatización, sino que en el contexto de lo público, se trata de asimilar las prácticas del sector privado, dado que se asumen como mejores, situación que lleva a que desde lo público se emulen las redes de prácticas de la empresa. Si bien hay una relación entre ambos aspectos marcados, el neoliberalismo económico, y la nueva gestión pública, su génesis y discursos no son los mismos, por lo cual no pueden ser homologadas. Lo que hubo en diferentes países de Latinoamérica fue una suerte de imbricación, donde se presentaron procesos de privatización fuertes, pero a su vez se incorporó en lo público la racionalidad de la empresa privada, donde la racionalidad definida desde el capital económico ganó terreno sobre el capital cultural, elementos que en lo público retóricamente tendría más peso.
} 
En términos de su gestión y de las prácticas institucionales se presenta lo que Galcerán denomina la Universidad-Empresa, la cual es resultado de la incorporación de la universidad a los circuitos empresariales y mercantiles de la sociedad capitalista actual (2010, p. 16). Sumado a la creciente desfinanciación de las universidades públicas, se les propone modelos gerenciales provenientes del mundo de la empresa, su orden de valores, sistemas de relevancias, lenguaje y taxonomías. ${ }^{8}$ En otras palabras, "si la universidad tiene que funcionar como una empresa privada, debe de gobernarse como una empresa privada" (Ferreiro, 2010, p. 133).

Otra forma de entender éste fenómeno, es mediante la distinción que hace Ball entre privatización exógena y endógena:

Mientras que el primero implica compañías privadas que se introducen en la educación para hacerse cargo de responsabilidades directas, servicios o programas, el último se refiere a cambios en el comportamiento de las propias organizaciones del sector público, que actúan como si fueran empresas, tanto en relación a los clientes y trabajadores como en los tratos con otras organizaciones del sector público (2007, p. 14. En Rivzi y Lingard, 2013, p. 162)

Desde la perspectiva de la nueva gestión pública, la privatización endógena se hizo presente, tornando esto en prácticas administrativas del mundo de la empresa privada.

Los cambios suscitados a nivel contextual, no sólo en Colombia, sino en Latinoamérica, conllevaron varias cosas para las universidades en términos de la incorporación de las nuevas reglas de juego de la economía neoliberal, por ejemplo, la métrica del monitoreo y la evaluación. Es así, que, señala Acosta

Las nuevas reglas que se derivaron de la instrumentación de las políticas, significó un proceso de "adaptación incremental" de las universidades el nuevo marco de políticas gubernamentales. La utilización de diversos recursos de poder por parte del gobierno para inducir cambios en las universidades, puso en movimiento diversas estrategias universitarias de adaptación a las nuevas reglas y condiciones de juego (Acosta, 2000, p. 35).

Uno de los efectos más fuertes para la "adaptación incremental" fue la adopción de formas de gestión y contables que respondieran a la dinámica que el contexto le iba exigiendo al campo universitario (y el económico).

En Colombia esto toma fuerza a partir de la expedición de la ley 30 de 1992 la cual no soluciona el problema de la desfinanciación gradual de las universidades públicas la cual persiste hasta hoy. Dicha situación, no desentonaba con los procesos que se venían dando en la educación en diversas latitudes, donde la educación se vinculaba cada vez más a circuitos empresariales.

\footnotetext{
${ }^{8}$ Dirá Vega al respecto: "La universidad empresarial se concibe como un negocio, que no se diferencia de ningún otro, en razón de lo cual la educación se convierte en una "industria", cuyo objetivo supremo radica en obtener un producto mercantil (un título), para lo cual existen insumos, que son los estudiantes, y administradores de esos insumos que son los profesores. Vistas así las cosas, el proceso educativo es un procedimiento que debe funcionar con todas las características que debe funcionar un proceso productivo, como quien dice se debe buscar la eficiencia, la eficacia, la productividad (rendimiento), la calidad, la excelencia del producto final" (2015, p. 190-191)
} 
Esta dinámica, manifiestan Girotto, Mundet, Llinás (2013) imponen una perspectiva gerencialista a las universidades, la cual surge para hacer frente al desafío competitivo que impone la economía a escala mundial. "El gerencialismo, en cuanto importante conjunto de tecnologías y prácticas, puede contemplarse como un producto de la intersección de las racionalidades de las políticas neoliberales y de las recetas de la gestión empresarial" (Girotto, Mundet, Llinás, 2013, p. 97).

Como parte de dicho proceso, se presenta lo que Vega llama una "razón instrumental de la productividad cuantitativa, que todo lo mide y lo reduce a cifras. De esta manera, se ha impuesto la lógica de las acreditaciones, revistas indexadas, rankings en los que se ubican a las instituciones, profesores, créditos y estudiantes" (2015, p. 10). Basta con revisar informes de gestión de las universidades para constatar dicha racionalidad del cálculo para dar cuenta de la gestión universitaria.

Cuantificación y productividad es vital en los procesos gubernamentales (en el sentido Foucaultiano) ${ }^{9}$, pues permite a partir de cifras orientar, evaluar y corregir las conductas, lo cual se traduce en los planes de desarrollo institucionales y planes de acción, tanto en medidas y clasificaciones que definen lo que es el ideal de universidad, a la vez que lo traduce en indicadores en cifras financieras, transmutando a lo financiero objetivos, metas y proyecciones, y es desde allí, desde el marco de lo económico y su nuevo protagonismo que se toman decisiones o se juzga lo acaecido.

Esta posición, siguiendo a Rose tiende a darle un poder a la cuantificación y su tinte de objetividad. Este poder de los números es tal que "se hacen invisibles y por tanto irrebatibles la compleja variedad de juicios y decisiones que entraña una medida, una escala, o un número" (1999, p. 208. En Rivzi y Lingard, 2013, p. 173).

A todo esto Rivzi y Lingard lo denominan "política como números" donde los indicadores de rendimiento, estadísticas, tablas de clasificación de medidas y demás, hacen parte de los procesos de planeación y control de las entidades públicas (2013, p. 159), entre ellas, las del subcampo de las universidades públicas. La gestión es evaluada a partir de los resultados expresados en datos cuantitativos, gastos, costos, porcentajes de cumplimiento y satisfacción ${ }^{10}$, los cuales son un mecanismo importante en el gobierno de las poblaciones, en este caso de quienes habitan la universidad.

Un ejemplo destacable atañe a los procesos de investigación. Dichas métricas "abarcan desde el número de artículos publicados y patentes desarrolladas, hasta la rapidez y profusión con que se han citado los primeros (o las revistas en que están publicados), o el rendimiento económico de las segundas" (Hortal, Magalhães, Pérez, Valladares, Santamaría, Escudero, Moya, 2018)11. Lo anterior también se expresa en la relevancia que

\footnotetext{
9 Para Foucault, la gubernamentalidad será entendida como como "el conjunto constituido por las instituciones, los procedimientos, análisis y reflexiones, cálculos y tácticas que permiten ejercer esta forma de ejercicio del poder que tiene por objetivo principal la población" (Foucault, 2007, p. 213).

${ }^{10}$ Como ejemplo de este discurso, puede verse la presentación que hace el Ministerio de Educación Nacional sobre Indicadores para la gestión escolar: "Lo que no es susceptible de medición no es posible de mejorar" (Ministerio de Educación Nacional, 2010) También puede observarse los informes de gestión de las universidades, donde se pueden identificar todos estos aspectos.

${ }^{11}$ Es importante señalar en los efectos derivados de la racionalidad de la métrica y de la evaluación de la eficiencia en la investigación científica en el mundo académico. Cabe resaltar el ambiente competitivo que se genera y a veces la racionalidad pragmática que se sintoniza con las ansias de la productividad. El conocimiento que de antaño ha sido un
} 
empiezan a tener los procesos de planeación, los presupuestos, los costos y la auditoría en la configuración de la delimitación de marcos de acción y redes de prácticas en las universidades.

\section{CAMBIOS EN LA CONTABILIDAD UNIVERSITARIA ANTE LA NUEVA RACIONALIDAD NEOLIBERAL}

En el caso de la planeación y los presupuestos, estos ganan importancia para la concreción de objetivos futuros, bajo métricas, acciones específicas, indicadores y asignación de recursos. El discurso de la Nueva Gestión pública señalará al respecto

Este nuevo sistema de dirección exige la elaboración de la estrategia o plan estratégico, donde se recoge la misión o propósito de la organización concretándola en las metas generales u objetivos a largo plazo necesarios para su consecución, así como el plazo marcado para alcanzarlos; y la planificación y programación anual, o plan operativo, con una fuerte vinculación al proceso de elaboración del presupuesto, que concreta las líneas de actuación a corto plazo cuya responsabilidad es del gestor público (García, 2007, p. 50-51).

La planeación y el presupuesto asociado a ella terminan siendo hermanos siameses, donde la primera (la planeación) marca un lindero, pero desde las condiciones de posibilidad que permite el segundo (las capacidades presupuestales y de financiación). El criterio financiero gana protagonismo como referente relevante para la toma de decisiones bajo la racionalidad empresarial. Esto no quiere significar que la planeación y el presupuesto sean de por sí guiados necesariamente por un fatalismo financiero ${ }^{12}$, sino más bien, que la racionalidad que los atraviesa los dota de sentido y los instrumentaliza hacia sus necesidades económicas en el contexto del neoliberalismo y de la universidad empresarial.

En otras palabras, el tamiz bajo el cual se toman decisiones ya no es el criterio netamente académico o social, en términos de aprobación de recursos para investigación, vinculación de docentes, bienestar universitario, entre otros, sino el uso eficiente de los recursos y la consecución de financiación vendiendo investigación y posgrados, arrendando instalaciones u ofreciendo servicios y mercancías a la comunidad, a las empresas o al Estado.

proceso colaborativo, se torna en un proceso que busca ser individualizado, destacando al individuo por encima de los procesos plurales. De esto que Hortal et. Al. (2018) plantee que "El uso generalizado de métricas simples ha propiciado que se asiente el cambio de ciencia colaborativa a ciencia competitiva. El ambiente competitivo es tal que entre los investigadores la producción científica ha llegado a convertirse en un espectáculo similar a la clasificación de la liga de fútbol o al ranking de tenistas en la ATP, al centrar la importancia de su labor más en el continente (dónde se publica y cuánto se cita) que en el contenido (cuánto ha avanzado el conocimiento). Según modelos matemáticos basados en procesos de selección natural, este tipo de ambiente competitivo genera ciencia de mala calidad incluso en colectivos de investigadores que siguen un código ético intachable. Y de hecho, los niveles elevados de competición son suficientes para reducir la calidad de las publicaciones científicas, menoscabar el potencial de hacer descubrimientos transformadores, incentivar la publicación de resultados poco fiables o difícilmente repetibles (como los llamados "falsos positivos"), y desincentivar la crítica al conocimiento ya establecido".

${ }^{12}$ Esto se puede evidenciar en el trabajo realizado con una comunidad campesina en el Oriente antioqueño donde la planeación y el presupuesto eran atravesados por una racionalidad no financiera y más por los intereses colectivos de los integrantes de la asociación de productores. Ver Cardona e Hinestroza (2013) La planeación y el presupuesto como sistemas simbólicos articulados a los procesos de resistencia campesina: análisis de una organización a partir de los referentes teóricos de Pierre Bourdieu. 
Otro aspecto relevante, es el hincapié que se hace en los resultados, los cuales además deben ser logrados de manera eficiente,

es decir, con el mínimo gasto posible. La eficiencia también ha sido asociada al discurso de la efectividad, que aquí significa el logro de metas y objetivos. Todo ello conlleva una nueva forma de dirigir la implementación de políticas y los resultados a través del establecimiento de objetivos y la creación de indicadores de rendimiento en relación a los objetivos (Rivzi y Lingard, 2013, p. 157 158).

\title{
IMPACTO DEL CAMBIO EN LA RACIONALIDAD ORGANIZACIONAL DE LO PÚBLICO CASO: Planeación de negocios y Los museos en Alberta Canadá
}

Por su parte en el caso de los Museos en Alberta Canadá, las restricciones presupuestales fueron un aliciente para que las entidades gubernamentales determinen como prerrogativa para la asignación de recursos la elaboración de planes de negocio que permitieran a los museos financiarse además con lo que se les asignaba, con recursos propios. Este cambio trajo consigo varios fenómenos. Por un lado, hubo un cambio significativo en términos del lenguaje utilizado para nombrar el acontecer del museo y las actividades del mismo, donde los estados financieros y herramientas de gestión ganan predominancia. Así mismo, hay un cambio en la preponderancia de los capitales, pues esta nueva dinámica relevó al capital cultural, y el capital económico ganó importancia. De la mano a esto, los historiadores, antropólogos, curadores, investigadores educadores perdieron peso en las decisiones de los museos, y administradores y contables ganaron terreno. Esto, dado que ahora estaba más enfocado hacia la generación de ingresos (Oakes, Townley y Cooper, 2009, p. 201).

Lo anterior supuso, cambios en aspectos simbólicos, taxonomías y lenguaje, implicó la incorporación del lenguaje financiero por parte de los diferentes integrantes de los museos. Los gerentes y demás integrantes del museo deben de verse como si trabajaran en un negocio. Dirán dichos los autores al respecto:

\begin{abstract}
La gestión ahora se centra en un proceso llamado "planeación de negocios". Cada vez más los gerentes sugieren que solamente a través de una articulación de las misiones, las metas, los objetivos y las estrategias, sus organizaciones tendrán una oportunidad de ser exitosas. Pero esas misiones, metas y objetivos no son suficientes. Además de esto, las organizaciones tienen que especificar con antelación los resultados visibles y medibles que quería conseguir y ofrecer evidencia que demuestre que en verdad los han conseguido. Como los resultados se definen por la generación de ingresos, tal evidencia requiere de pruebas que demuestre que los sitios están elaborando productos que se puedan traducir en capital económico (Oakes, Townley y Cooper, 2009, p. 201)
\end{abstract}

De Allí, que la contabilidad financiera gane terreno como parte de los procesos de gestión, como de la rendición de cuentas. Este proceso implicó, como se ha señalado, múltiples cambios simbólicos, en las diferentes posiciones de los agentes en los museos, como en general en un cambio de racionalidad, donde los aspectos que definían el quehacer institucional se transforman cuando el capital económico y la preponderancia de lo financiero ganan terreno. Finalmente, los autores concluirán señalando estos cambios implicaran que

\begin{abstract}
Los directivos se sintieran forzados u obligados a participar en el proceso de planeación de negocios. Ellos son llamados a enfocarse en las categorías del plan de negocios y de esta manera, deben cambiar su identidad como productores y así menoscabar una característica que es la base de su capital cultural. Es en este sentido que la planeación de negocios ejerce su control en la forma de violencia simbólica (Oakes, Townley y Cooper, 2009, p. 209).
\end{abstract}

La articulación de lo contable a dicha racionalidad se torna en un elemento relevante que permite hacer seguimiento a las acciones de los sujetos, pero también da nuevas taxonomías para evaluar y proyectar el acontecer organizacional. Esos nuevos referentes simbólicos se convierten en mecanismos de cognición y posibilitan un lenguaje común para comprender en entramado de vínculos que supone la dinámica organizacional, pero también se configura en fuente de motivaciones y alicientes para el actuar prospectivo de las entidades. 
Pero esta racionalidad sería insuficiente sin sistemas de seguimiento que permitan tomar medidas meticulosas sobre las acciones, los recursos que circulan y se invierten en la organización. De allí la relevancia que tienen los sistemas de costos y en general la contabilidad de gestión en la toma de decisiones. La racionalidad de la eficiencia y la eficacia desde criterios financieros no puede ser ajena a la contabilidad de gestión que se materializa por ejemplo en la implementación cada vez más frecuentes de sistemas de Costeo Basados en Actividades ${ }^{13}$ ( $A B C$ en sus siglas en inglés) y software que cohonesten con esto como por ejemplo el SAP (Systeme Anwendungen und Produkte palabra alemana que se traduce como 'sistemas, aplicaciones y productos') que gana juega un papel importante en la administración pública ${ }^{14}$. Como dirá David Westbury (1997) en la década de los 90 "un adecuado costeo para la toma de decisiones en universidades es vital, en especial cuando existen restricciones de presupuesto" (En Del Carpio, 2007, p. 26).

En este sentido, dirá Costa (2012) "El ABC no es solamente un método de cálculo del costo de producción, sino también un método de control de gestión. Este provee información contable útil y fundamental para reflejar el posicionamiento competitivo de la empresa" (p. 530). Afirmación que cobra sentido bajo los estándares de competitividad entre las universidades en el sistema de rankings y clasificaciones nacionales e internacionales.

En esa misma dirección, López et al (2011) indica que

La razón de querer disponer de los costes de las actividades universitarias es doble. De una parte son un instrumento de gestión interna, algo que las universidades necesitan para controlar a los responsables de las misiones asignadas (vicerrectorados, servicios, departamentos, centros...) e instrumentos de información externa, para producir indicadores que demuestren a los grupos sociales interesados la productividad de los recursos que les han sido confiados (p. 23).

Como se ha insistido, los conceptos de eficiencia (económica) y productividad terminan siendo referentes relevantes para la gestión, y en este caso los sistemas de costos logran convertirse en un conocimiento que torna visible el acontecer de la organización desde unos lentes específicos. El coste de los procesos, el costo unitario por estudiante, por facultades, de los productos y servicios es concomitante con la racionalidad financiera. "La información proporcionada por el sistema $A B C$ ayuda a tomar decisiones sobre fijación de precios, subcontratación externa, gastos de capital y eficiencia operacional" (Costa, 2012, p. 535).

Los costos se tornan en un buen espectrómetro para el seguimiento y la vigilancia. El ojo de la contabilidad de gestión se posa sobre las acciones, sobre los procesos, y desde luego permite la regulación de las conductas, que en el marco de un régimen de la eficiencia deben de contenerse ante aquello que propicie el derroche de tiempo y de

\footnotetext{
${ }^{13}$ Es importante resaltar las implicaciones que esto tiene en las relaciones de poder y en los espacios de visibilidad el gobierno de sí y de los otros. Muy bien lo señalaban Miller, P., \& O’Leary, T. (2009) la contabilidad de gestión, y en particular de costos ayudan a construir a personas gobernables.

${ }^{14}$ Con este software la contabilidad irradia en todos los procesos de las distintas dependencias y las unidades académicas que son quienes deben alimentar el sistema. Esto dinamiza prácticas que son contables en los diferentes niveles de acción de la universidad.
} 
recursos. De esto, que los costos no propicien sólo un sistema de información, sino que también posibilite la regulación de las conductas.

De manera congruente, los procesos de presupuestación, de costeo van de la mano indefectiblemente con la "cultura de la auditoría", de la evaluación de resultados, los controles y la gestión del riesgo ${ }^{15}$. Todo esto, de la en concordancia con el énfasis que le da la nueva gestión pública a la eficiencia, a la efectividad y a la supervisión constante de éstas (Rivzi y Lingard, 2013, p. 161).

De esto, que los procesos de planeación, presupuestación, costeo deban de tener un seguimiento juicioso desde los procesos de control interno, gestión del riesgo y auditoría, pues como elemento de control permanente y posterior, las prácticas son evaluadas según los propósitos organizacionales, demarcados en el espíritu estratégico y prospectivo de la entidad. En este caso, no se trataría sólo de hacer seguimiento a posibles malversaciones de fondos, sino de controlar el rumbo, y tomar los correctivos para el cumplimiento de los objetivos y metas propuestos bajo los criterios, como se ha insistido, de eficiencia, eficacia y productividad. La auditoría, como los presupuestos, estándares y otros indicadores aparentemente tan triviales, son cruciales para la operacionalización de programas de gobierno a distancia que caracterizan las formas de la nueva administración pública (Rose, O’Malley y Valverde, 2012, p. 133).

Como se puede evidenciar, los cambios en los procesos de gestión implican cambios en las formas de gobierno en las universidades, y no es un asunto nimio, pues atraviesa todo el campo de la universidad, dinamiza ciertas prácticas en detrimento de otras, a la vez que gesta un sistema de percepción para dar sentido a la realidad y actuar ante ella, desembocando en un conjunto de acciones, programas, proyecciones y sistemas de control acordes a los nuevos retos del campo de las universidades.

Finalmente, cabe señalar que en el marco de estas transformaciones de la racionalidad de la gestión universitaria, los presupuestos, los costos y la auditoría adquieren unos sentidos concretos. Desde una perspectiva diferente habría que tener una racionalidad menos financiera en las universidades, de tal forma que los sistemas de presupuestos, costos y auditorías jueguen roles diferentes, en dicho caso, el eje articulador ya no sería la racionalidad estratégica financiera. Lo anterior posibilitaría otras acciones encaminadas a acrecentar el capital cultural de las universidades, la dignificación del trabajo docente, a investigaciones sin ánimo de lucro y conectadas con el bienestar social y ambiental, por dar algunos ejemplos.

En correlato, los cálculos ya no serían financieros, y habría que buscar otras formas de rendición de cuentas, de toma de decisiones y de control que desde una lógica financiera permitan evaluar esos aspectos que se quedan en la sombra desde la racionalidad económica. No se trata entonces de sacar de plano a la contabilidad, sino más bien posibilitar mecanismos para que ésta desde una racionalidad estratégica diferente se incorpore a dispositivos que dinamicen la organización universitaria a objetivos más sociales y académicos, y menos financiarizados.

\footnotetext{
15 Un ejemplo de esto está asociado a la adopción de modelos de control interno como el MECl (Modelo estándar de control interno) para el sector público y en particular para las universidades, donde se insiste entre otros aspectos en incorporar en la comunidad universitaria una cultura del control. Como se dirá en una publicación de la Universidad de Antioquia ";El Modelo Estándar de Control Interno nos compete a todos! (Universidad de Antioquia, 2017).
} 


\section{CONCLUSIONES}

El campo de las universidades ha venido transformándose a raíz de su relación cada vez más cercana con el campo económico, lo cual va de la mano con los procesos que se gestan a nivel global. Las redes cada vez más complejas que atraviesan a las universidades y la interpelan a cambiar para responder a las nuevas realidades han llevado a que la racionalidad que direcciona la universidad mute.

Nuevas formas de gestión y de contabilidad se acoplan a la lógica que acoge la racionalidad empresarial al ámbito de lo público, y que lidera los procesos de toma de decisiones en las universidades.

Tanto el lenguaje empresarial como sus criterios para tomar decisiones ganan terreno en la universidad, que en el caso de la universidad pública va de la mano con la desfinanciación y los imperativos cada vez mayores para la consecución de recursos para garantizar su continuidad en el tiempo.

Para lo anterior, la propuesta de la Nueva Gestión Pública y la racionalidad neoliberal terminan siendo una prerrogativas para las universidades que instalan un conjunto de dispositivos que permiten gobernar los destinos de la universidad desde criterios de la empresa privada.

La nueva racionalidad que se instala en las universidades conlleva necesariamente prácticas que incluyen un fuerte componente contable, donde el funcionamiento organizacional se ve mediado por un conjunto de conocimientos y técnicas que dan sustento la lógica empresarial que la racionalidad de la nueva gestión pública encarna.

Dicha racionalidad empresarial se torna en el elemento dinamizador de prácticas de gestión y lenguaje corporativo, donde la eficiencia, la eficacia y la productividad ganan terreno. De allí que los procesos de planeación, presupuestos, costos, auditoría, control interno y gestión de riesgos se vinculen a la plétora de saberes que confluyen para dar sustento a prácticas que a modos de dispositivos de gobierno buscan dirigir las conductas, no necesariamente de un modo disciplinario, sino desde el poder gubernamental, en el sentido dado por Michel Foucault.

En palabras de Castro-Gómez este tipo de gobierno gubernamental

consiste en conducir conductas, es decir, en poner en marcha un conjunto de acciones sobre acciones posibles: incitándolas, induciéndolas, desviándolas, facilitándolas o dificultándolas, haciéndolas más o menos probables. En el límite, también prohibiéndolas, pero teniendo siempre en cuenta la libertad (las múltiples acciones posibles) de aquellos a quienes se busca conducir y su capacidad de actuar sobre quienes los conducen (2014, p. 108).

Y en la línea argumentativa que se viene manejando, la contabilidad y la auditoría como tecnologías de gobierno, permiten actuar sobre las acciones, vigilando, midiendo, valorando, visibilizando, orientando las decisiones. De allí que en la red de prácticas en que lo contable se articula, el saber adquiera un sentido práctico para gobernar la universidad, a su población. 
La comprensión de la contabilidad derivada de los argumentos que se vienen presentando, permite evidenciar que no hay unos preceptos que definan a la contabilidad, no hay un ser per se de la contabilidad, sino que éste existe en relación a las redes de prácticas que se establecen en las organizaciones y la racionalidad estratégica que dinamiza cierto tipo de vínculos y de redes de relaciones. Muy bien podría actuar la contabilidad desde una racionalidad no neoliberal en la universidad, donde los criterios de eficiencia, eficacia y productividad económica fueran contingentes y el peso en lo académico y social se situaran como criterios que dirigieran el accionar de la comunidad universitaria.

Finalmente, estos sistemas de rendición de cuentas, lejos de ser algo nimio, resaltan el potencial activo de la contabilidad, y su protagonismo como parte de unos dispositivos que articulan prácticas en función de una racionalidad y un sentido estratégico económico, que pone el acento en lo financiero.

Es en ese entramado de relaciones, en esas redes de prácticas que debe ser pensada la contabilidad, pues su solvencia, pero también sus implicaciones traspasan lo técnico, y se juegan en el vínculo de las relaciones de poder que atraviesan las organizaciones, y en este caso, el sentido de lo contable y la rendición de cuentas no puede ser un a priori, sino el producto del conocimiento concreto de las dinámicas organizacionales.

\section{REFERENCIAS}

Acosta, A. (2000) Estado, políticas y universidades en un periodo de transición. México: Fondo de Cultura Económica.

Audelo, B. (2013) La evaluación de la educación superior. Un estudio comparativo: México y Colombia. Juan Pablos Editores, Universidad de Sinaloa. México.

Barrios, C. y Rivera, Y.A. (2008). El papel de los sistemas contables administrativos (SCA) en la ordenación espacio-temporal de las prácticas sociales en las organizaciones modernas. Contaduría Universidad de Antioquia, 52, 61-84. Disponible en http://aprendeenlinea.udea.edu.co/revistas/index.php/cont/article/view/2158

Bourdieu, P. (2001) Contrafuegos 2. Por un movimiento social europeo. Barcelona: Anagrama.

Bourdieu, P. (2013) Las estrategias de la reproducción social. Buenos Aires: Siglo XXI editores.

Cardona, J. \& Hinestroza, Y. (2013) La planeación y el presupuesto como sistemas simbólicos articulados a los procesos de resistencia campesina: análisis de una organización a partir de los referentes teóricos de Pierre Bourdieu. Revista Activos, 20, pp. 55-100

Castro, E. (2014) Introducción a Foucault. Argentina: Siglo XXI editores.

Castro-Gómez, S. (2010) Historia de la gubernamentalidad. Razón de Estado, liberalismo y neoliberalismo. Bogotá: Siglo del hombre.

Chica, S. (2011). Una mirada a los nuevos enfoques de la gestión pública. Administración \& Desarrollo $\quad 39 \quad$ (53): $\quad 57-74 . \quad$ Disponible http://datateca.unad.edu.co/contenidos/109133/Documentos/Una mirada a los nuevos enfoq ues de la gestion publica.pdf

Costa, M. (2012). Contribución del modelo ABC en la toma de decisiones: el caso universidades. Cuadernos de Contabilidad, 13 (33), 527-543. 
Del Carpio, J. (2007) Aplicación del costeo basado en actividades en las Universidades. Industrial Data. 10(2), pp. 26-29. Disponible en http://www.redalyc.org/articulo.0a?id=81620574004

Feldfeber, M (2009) Educación "¿En venta?" Tratados de libre comercio y políticas educativas en América Latina. En Gentili, P., Frigotto, G., Leher, R. \& Stubrin, F. (Ed.) Políticas de privatización, especio público y educación en América Latina (pp. 135-161). Rosario, Argentina: Homo Sapiens Editores.

Ferreiro, X. (2010) Mercantilización y precarización del conocimiento: El proceso de Bolonia. En EduFactory \& Universidad Nómada. La universidad en conflicto. Capturas y fugas en el mercado global del saber. Madrid: Traficantes de sueños.

Foucalt, M. (2007) La "gubernamentalidad". En Ensayos sobre biopolítica: excesos de vida. Giorgi, G.; Rodríguez, F.; Foucault, M.; Deleuze, G.; Agamben, G.; Negri, A; Zizek, S. Argentina: Paidós. Disponible en https://programaddssrr.files.wordpress.com/2013/05/ensayos-sobrebiopolc3actica.pdf

Foucault, M. (2008). Nacimiento de la biopolítica. Buenos Aires: Fondo de Cultura Económica.

Galcerán, M. (2010) La educación universitaria en el centro del conflicto. En Edu-Factory \& Universidad Nómada. La universidad en conflicto. Capturas y fugas en el mercado global del saber. Madrid: Traficantes de sueños.

García, I. (2007) La nueva gestión pública: evolución y tendencias. Presupuesto y Gasto Público (47) p.p. 37-64 Disponible en http://www.ief.es/documentos/recursos/publicaciones/revistas/presu_gasto_publico/47_GarciaS anchez.pdf

Girotto, M., Mundet, J. y Llinàs, X. (2013) Estrategia en la universidad: ¿cuestión de calidad, gerencialismo y relaciones político-financieras? Revista de Educación (361) pp. 95-116 Disponible en $\quad$ http://www.mecd.gob.es/dctm/revista-deeducacion/articulosre361/re36104.pdf?documentld=0901e72b8162f02d

Hortal, J., Magalhães, S., Pérez, S., Valladares, F., Santamaría, L., Escudero, A. \& Moya, J. (14 de febrero de 2018) El uso excesivo de métricas y la lógica capitalista pervierten el trabajo científico. Eldiario.es. Recuperado de https://www.eldiario.es/cienciacritica/excesivo-metricas-capitalistapervierten-cientifico 6 740235988.html

López, F. (2009) La universidad latinoamericana y los procesos de internacionalización: Estrategias de financiamiento. En Gentili, P., Frigotto, G., Leher, R. \& Stubrin, F. (Ed.) Políticas de privatización, especio público y educación en América Latina (pp. 239-264). Rosario, Argentina: Homo Sapiens Editores.

López, A. (2011) Libro blanco de los costes en las universidades. Madrid: Editorial Evergráficas, S. L.

Lawrence, A., Northcott, L. (1997). Accounting systems and systems of accountability in the New Zealand health sector. Accounting, Auditing \& Accountability Journal, 10(5), 665-683.

Lotta, R. (2004). "La globalización imperialista y la lucha por un futuro diferente". En: varios autores, El punto de vista marxista sobre la globalización. [s.e.], Bogotá.

Martínez, J. (2014) Subjetividad, biopolítica y educación: una lectura desde los dispositivos. Bogotá: Ediciones Universidad de la Salle.

Ministerio de Educación Nacional (2010) Lo que no es susceptible de medición no es posible de mejorar. Disponible en https://es.slideshare.net/alexmema/presentacion-no-4-metas-eindicadores3 
Miller, P. y O'Leary, T. (2009). La contabilidad y la construcción de la persona gobernable. En Gómez, M. y Ospina, C. (Eds.). Avances interdisciplinarios para una comprensión crítica de la contabilidad: Textos paradigmáticos de las corrientes heterodoxas (pp. 175-217). Medellín: Escuela de Administración y Contaduría Pública - Universidad Nacional de Colombia y Departamento de Ciencias Contables - Universidad de Antioquia.

Múnera, L. (2005) ¿Hacia dónde va la universidad pública? Tendencias globales en política pública para la educación superior. Disponible en http://firgoa.usc.es/drupal/node/20705

Núñez, J. (2011) La genealogía como filosofía política en Michel Foucault. México: Plaza y Valdez editores.

Oakes, L., B. Townley, y D. Cooper. (2009) La planeación de negocios como pedagogía: Lenguaje y control en un campo institucional cambiante. En M. Gómez, y C. Ospina, (eds.) Avances interdisciplinarios para una comprensión crítica de la contabilidad: Textos paradigmáticos de las corrientes heterodoxas (pp. 175-217). Medellín: L. Vieco e hijas Ltda.

Orlenas, J. (2009) Neoliberalismo y capitalismo académico. En Gentili, P., Frigotto, G., Leher, R. \& Stubrin, F. (Ed.) Políticas de privatización, especio público y educación en América Latina (pp. 83-120). Rosario, Argentina: Homo Sapiens Editores.

Pelayes, O (2000) La hipocresía neoliberal: las nuevas formas de privatización de la educación como utopía democratizadora. Revista Herramienta, 12. Disponible en http://www.herramienta.com.ar/revista-impresa/revista-herramienta-n-12

Rizvi, F. y Lingard, B. (2013). Políticas Educativas en un Mundo Globalizado. Morata: Madrid.

Rose, N., O'Malley, P. y Valverde, M. (2012) Gubernamentalidad. Astrolabio (8) p.p. 113-152. Disponible en https://revistas.unc.edu.ar/index.php/astrolabio/article/viewFile/2042/1037

Sauquillo, J. (2001). "Michel Foucault: una insurrección de los saberes sometidos". En: Máiz, Ramón (comp.).Teorías políticas contemporáneas. Valencia: Tirant lo Blanch, pp. 61-90.

Santos, B. (2007) La universidad en el Siglo XXI. Para una reforma democrática y emancipadora de la universidad. La paz, Bolivia: Plural Editores. Recuperado de http://www.boaventuradesousasantos.pt/media/universidad siglo xxi-.pdf

Sibilia, P. (2005) El hombre postorgánico. Cuerpo, subjetividad y tecnologías digitales. México: Fondo de Cultura Económica.

Soforcada, F. (2009) Alambrando el bien común: conocimiento, educación y derechos sociales en los procesos de privatización y mercantilización de las últimas décadas. En Gentili, P., Frigotto, G., Leher, R. \& Stubrin, F. (Ed.) Políticas de privatización, especio público y educación en América Latina (pp. 135-161). Rosario, Argentina: Homo Sapiens Editores.

Universidad de Antioquia (2017) ¡El Modelo Estándar de Control Interno nos compete a todos! Disponible

http://www.udea.edu.co/wps/portal/udea/web/generales/interna/!ut/p/zO/rY7LDolwFES XQVL Oyso4rKpxkCIC1 RbkyFBat9gK3Gz7cYTXXs3d0zdzlziKINoppdRcWcMJpJz1sa75IRCXu4D I0nhHA McGT4WKdh0mIMkTfDTBLx94QrcgyzQEgahPEsWkoRrQw2vGbQ5vanB2TI5KzAJj9pINR HVbo4 xt4bvmd8dfagLgapaclca2-iNliydJYZ3 BKB4lbrOeBnVJ7ofyKpzBzECpmg!/

Vega, R. (2015) La universidad de la ignorancia. Ocena sur, Colombia 